\title{
E. Ann Ellis: A Life Dedicated to STEM/STEAM Outreach
}

Carol B. Johnson ${ }^{1}$, Zhiping Luo ${ }^{2}$, Hansoo Kim³ ${ }^{3}$, Richard Littleton ${ }^{3}$, Tom Stephens ${ }^{3}$, Michael Pendleton ${ }^{3}$, Christos Savva ${ }^{4}$, Stanislav Vitha ${ }^{3}$, Andreas Holzenburg ${ }^{5}$

${ }^{1 .}$ Department of Biology, Lonestar College, Houston, TX 77073, USA

2.Department of Chemistry and Physics, Fayetteville State University, Fayetteville, NC 28301

${ }^{3}$ Microscopy and Imaging Center, Texas A\&M University, College Station, TX 77843-2257 USA

${ }^{4}$.Leicester Institute of Structural and Chemical Biology, University of Leicester, Leicester LE1 7RH, United Kingdom

${ }^{5}$.Department of Biomedical Sciences, School of Medicine, The University of Texas Rio Grande Valley, Harlingen, TX 78550, USA

E. Ann Ellis, a consummate professional and member of the research staff at the Microscopy and Imaging Center at Texas A\&M University from 2002 to 2013, combined microscopy theory and praxis into an inspiring, and holistic teaching approach. An approach that provided undergraduate, graduate and high school students with the opportunity to be intricately involved in a project from its inception to completion, all while learning the steps necessary to accomplish the task at hand as well as being endowed with laboratory safety knowledge (Fig. 1). In addition to combining theoretical and practical aspects of microscopy, students learned about the contextual linkage of complex subject matter and learning how to learn.

From a didactic point of view, a focus on educational attainment through the use of microscopy was of particular importance to Ann. To this end, the principles of mentorship and inspiration were the cornerstones of the approach. Inspiration fuels creativity, which is of significance because our society is in the midst of preparing for the fourth industrial revolution [1]. Microscopy dovetails these two principles and facilitates educational opportunities that provide vision to students, particularly those of lowsocioeconomic status who have limited educational resources and limited educational exposure. For example, microscopy combines optics, vacuum systems, electronics, with chemistry and mathematics (i.e. image analysis/processing) thus embracing the spectrum of STEM education and teaching transferrable skills such as image composition. The latter also relates to the arts (i.e. STEAM).

Micrographs prepared in the laboratory were the inspiration for a learning experience with high school students through an outreach program termed the NSF Graduate Teaching Fellows in K-12 Education (GK-12) Program. This program provided opportunities for a GK-12 Fellow and a teacher to collaborate on ways to incorporate real-life content and application in the classroom. Alternative high school students were one of the targeted populations served by this program. Micrographs were used to introduce students to the scientific community, teach about molecular biology, and to foster critical thinking skills. Micrographs showing the ultrastructure of chloroplasts were used during a photosynthesis lesson. Studies have shown that microscopic images can be employed to enhance students' knowledge of biological concepts and that TEM and SEM micrographs are useful teaching tools [1-2]. These images provided a visual framework of the ultrastructure of photosynthetic organelles and sparked a conversation about molecular biology. The micrographs also introduced students to immunolabeling by relating how the presence of 12-nm colloidal gold particles corresponded to the presence of a specific protein in its immediate vicinity. As a result of the students' interest indicated by the overwhelming number of microscopy-related questions, a group visited the Microscopy and Imaging Center (MIC) at Texas A\&M University. There they received hands-on experience in imaging using TEM equipment and had the 
opportunity to ask the questions that were generated in the classroom prior to their on-campus visit (Fig. 2).

Ann's legacy lives on in the many students that she trained, inspired and mentored throughout her life. The first author on this abstract speaks for many of these students when stating that it was an honor and a privilege to be taught by one of the best staff microscopists in the field.

References:

[1] Klaus Schwab in "The Fourth Industrial Revolution, World Economic Forum”, (Crown, New York) p. 8.

[2] J. Wandersee in "The Content of Science: A Constructivist Approach to Its Teaching and Learning”, ed. P. Fensham, (Falmer Press, New York) 1994.

[3] P.J. McMillan, New Anat. 265 (2001) p. 222.

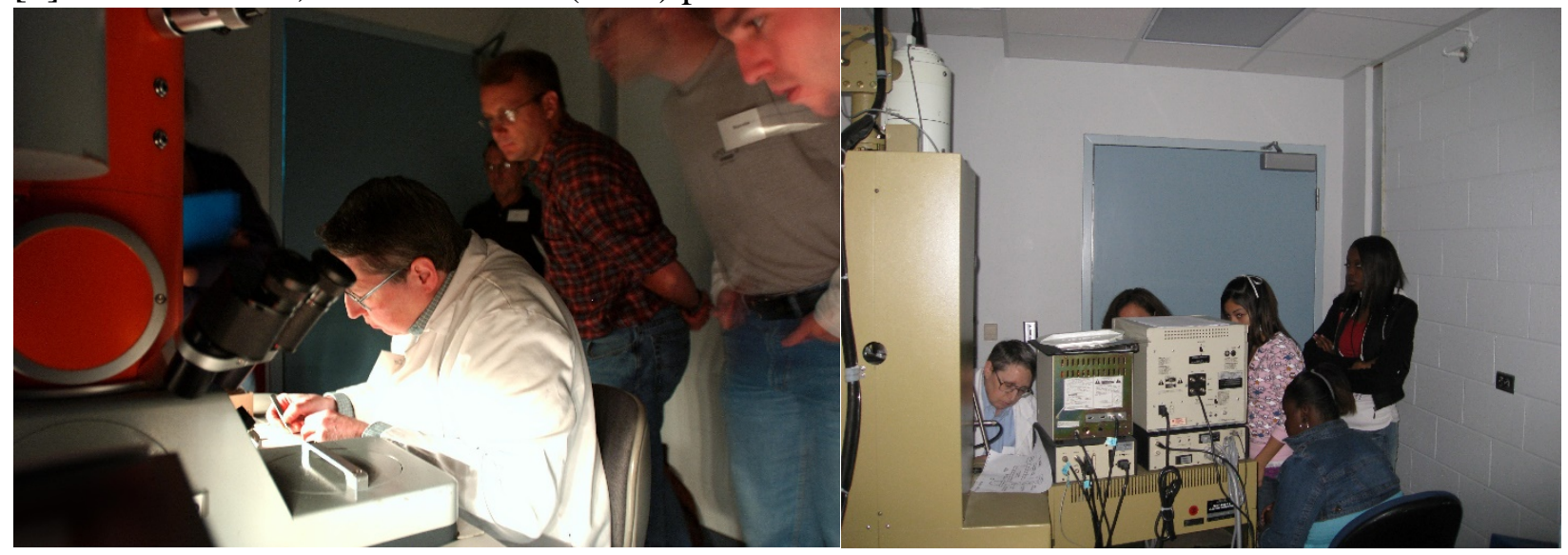

Figure 1. Sample Analysis. Students of different ages at the Texas A\&M MIC.

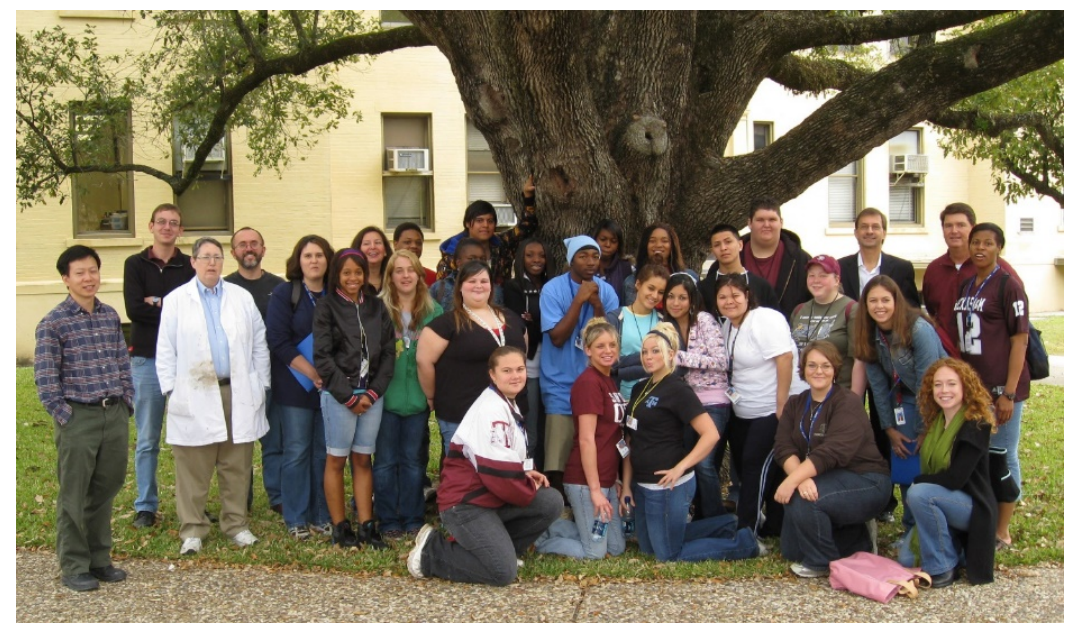

Figure 2. Microscopy Outreach. Trainers and trainees: oak tree (Quercus virginiana Mill. x lyrata Walt.) aftermath. 